\title{
Avaliação clínica e laboratorial do gel da Uncaria tomentosa (Unha de Gato) sobre candidose oral
}

\section{Leonardo Costa de Almeida Paiva, ${ }^{* 1}$ Rodrigo Alves Ribeiro, ${ }^{1}$ Jozinete Vieira Pereira, ${ }^{1}$ Neuza Maria Cavalcante Oliveira ${ }^{2}$}

\author{
${ }^{1}$ Faculdade de Odontologia, Universidade Estadual da Paraíba, Av. das Baraúnas 351, Campus Universitário, \\ Bodocongó, 58109-753 Campina Grande-PB, Brasil, \\ ${ }^{2}$ Faculdade de Farmácia, Universidade Federal da Paraíba, Cidade Universitária, 58051-900 \\ João Pessoa-PB, Brasil
}

\begin{abstract}
RESUMO: Na odontologia, a fitoterapia já vem sendo utilizada com sucesso há vários anos. Tratase de um meio terapêutico que apresenta como vantagem sobre as medicações alopáticas o fato de apresentar reações adversas mínimas. A Uncaria tomentosa é uma planta indígena da floresta Amazônica e de outras áreas tropicais da América do Sul e Central. Tem aplicação no tratamento de diversas patologias, entre elas a candidose. Este trabalho avalia clínico e laboratorialmente a ação do gel da Uncaria tomentosa em pacientes portadores de candidose na cavidade oral. Foram selecionados 20 pacientes que apresentaram clínico e laboratorialmente infecção pelo Candida. Os mesmos foram divididos em 2 grupos. O grupo-teste (Uncaria tomentosa/Imuno-Max Gel), composto por 10 pacientes, foi orientado a utilizar o gel da Uncaria tomentosa, sobre as lesões na cavidade oral, $3 \mathrm{x}$ ao dia por um período de 14 dias. O grupo-controle (Miconazol/Daktarin Gel) utilizou a medicação da mesma forma prescrita para o grupo-teste. Após o período de tratamento, os pacientes retornaram para nova avaliação clínica e laboratorial. A Uncaria tomentosa mostrou ser um fitofármaco promissor na odontologia, apresentando vantagem sobre o miconazol de não ter provocado reações adversas nos pacientes, uma vez que, $40 \%$ dos pacientes do grupo-controle, apresentaram reações indesejáveis.
\end{abstract}

Unitermos: Candidíase, Uncaria tomentosa, miconazol.

\begin{abstract}
Clinical and laboratorial evaluation of Uncaria tomentosa (Cat's Claw) gel on oral candidiasis". In dentistry, the phytotherapy is already being used successfully for some years now. It is about a promising therapeutical way in the pharmaceutical field, having as advantage on pharmacotherapy medications the fact to present minimum adverse reactions. The Uncaria tomentosa is an aboriginal plant of the Amazonian forest and other tropical areas of the South and Central America. It has application in the treatment of several pathologies, including candidiasis. This work evaluates, clinical and laboratorial, the action of the Uncaria tomentosa gel in the oral cavity candidiasis patients. Twenty patients which presented clinical and laboratorial signs of Candida infection were selected. They were divided in 2 groups. The test-group (Uncaria tomentosa/IMUNO-MAX Gel), with 10 patients, was told to use the Uncaria tomentosa gel, on the oral cavity injuries, 3 times a day for a period of 14 days. The control-group (Miconazol/ DAKTARIN Gel) used the prescribed medication in the same way of the test-group. After the treatment period, the patients returned for a new clinical and laboratorial evaluation. The Uncaria tomentosa showed to be a promising phytotherapeutical medication in dentistry, in the field of the anti-fungi treatment, presenting as advantage on the Miconazol not causing adverse reactions in the patients, once $40 \%$ of the control-group patients showed undesirable reactions.
\end{abstract}

Kaywords: Candidiasis, Uncaria tomentosa, miconazole.

\section{INTRODUÇÃO}

A infecção pelo Candida, um microorganismo fúngico semelhante à levedura, é denominada de candidíase, ou como preferem os ingleses, candidose (Neville et al., 2004). Entre os fatores predisponentes para a candidose, têm-se: próteses intra-orais, deficiências nutricionais, doenças metabólicas, drogas antibacterianas, deficiência orgânica das glândulas salivares e imunossupressão (Kleinegger et al., 2001). Em pacientes portadores de aparelhos protéticos, é comum o surgimento de estomatite protética quase sempre associada à presença de candidose eritematosa (Batista et al., 1999). Pacientes infectados com o HIV (vírus da imunodeficiência adquirida), apresentam uma gama de manifestações orais, muitas destas, refletem imunodeficiência. A candidose de orofaringe é a manifestação mais comum (Hunter et al., 1998). 
O diabetes mellitus é uma patologia sistêmica que pode causar várias anormalidades nos tecidos orais, entre elas à infecção por Candida (Guggenheimer et al., 2000; Moore et al., 2000; Neville et al., 2004). Em crianças com síndrome de Down, além das alterações anátomo-fisiológicas bucais, fatores adicionais as tornam mais suscetíveis a processos infecciosos, inclusive fúngicos, onde as espécies de Candida são os agentes etiológicos mais preponderantes (Vieira et al., 2005).

Pacientes com Síndrome de Sjogren apresentam mais predisponibilidade de desenvolverem a candidose, visto que as funções de limpeza, lubrificação e antibacteriana da saliva estão abaixo do nível normal (Radfar et al., 2003).

A radioterapia e a quimioterapia são considerados efetivos meios de controle e cura de neoplasias de cabeça e pescoço. Infelizmente, efeitos colaterais existem, entre eles a xerostomia, que leva a colonização ou infecção por espécies de Candida, principalmente a C. albicans.

As três principais classes de antifúngicos usados, atualmente, no tratamento da candidose são os polienos (como nistatina e anfotericina B), os imidazóis (como clotrimazol e miconazol) e os triazóis (como o fluconazol e itraconazol).

Geralmente, o tratamento inicial é feito com um agente tópico. Nistatina e miconazol são as drogas de escolha inicial. Caso a terapêutica tópica não apresente resultados, é iniciado o tratamento sistêmico, sendo o fluconazol a droga mais prescrita.

É preocupante a crescente resistência dos fungos às drogas disponíveis no mercado, havendo uma alta taxa de recidivas, o que faz com que novas drogas devam ser pesquisadas e avaliadas quanto a sua eficácia no tratamento antifúngico (Hunter et al., 1998; Patton et al., 2001; Patton \& Shugars, 2003).

$\mathrm{Na}$ odontologia, a fitoterapia já vem sendo usada com sucesso há vários anos. Trata-se de um meio terapêutico promissor no campo farmacológico, uma vez que tende a apresentar reações adversas bem menores e de intensidade diminuta em relação às medicações alopáticas (Oliveira et al., 2007; Silva et al., 2008; Vasconcelos et al., 2008).

A Uncaria tomentosa é uma planta indígena da floresta Amazônica e de outras áreas tropicais da América do Sul e Central (Martino et al., 2006). Os estudos científicos dessa planta foram iniciados nos anos 70, quando Klaus Keplinger, um jornalista e etnologista independente de Innnsbruck, Áustria, organizou o primeiro trabalho definitivo com a Uncaria tomentosa (Taylor, 2002).

A Uncaria tomentosa tem aplicação no tratamento das artrites, reumatismo, abscessos, inflamações, febre, alergia, neoplasias, úlcera gástrica, contracepção, irregularidade menstrual, recuperação de parto, impurezas da pele, diabetes, disenteria, dores de cabeça, infecções bacterianas e fúngicas, ação analgésica e sedativa (Heitzman et al., 2005; Jurgensen et al., 2005; Kloucek et al., 2005; Sheng et al., 2005; Akesson et al., 2005; Pilarski \& Skib; Gulewicz, 2006).

Nesta pesquisa foi utilizado o gel já disponível comercialmente e que se apresenta na concentração de $50 \mathrm{mg} / \mathrm{g}$ de extrato de Uncaria tomentosa, dispondo na sua formulação, no mínimo, $3,3 \mathrm{mg} \%$ de alcalóides totais, calculado como mitrafilina, este é um alcalóide oxíndole pentacíclico que tem propriedades imunoestimulantes e antiinflamatórias, essenciais no tratamento da candidose.

Este trabalho avalia clínico e laboratorialmente a ação do gel da Uncaria tomentosa em pacientes portadores de candidose na cavidade oral.

\section{MATERIAIS E MÉTODOS}

O estudo é do tipo quantitativo, observacional, transversal e descritivo. O universo foi constituído de pacientes, com faixa etária média de 44,35 anos, que procuraram atendimento na Clínica Integrada de Odontologia da Universidade Estadual da Paraíba. Foi utilizada ficha clínica para coleta de dados de identificação, anamnese dos pacientes, análise micológica, análise clínica e terapêutica.

A amostra foi constituída por 30 pacientes que foram selecionados após os resultados do exame clínico, quando os mesmos apresentaram sinais clínicos sugestivos de infecção por Candida. Foi realizada a coleta de material da mucosa oral, onde existia evidência clínica sugestiva de infecção. Em seguida o material foi encaminhado ao laboratório para fins de análise micológica confirmatória. Após a confirmação positiva, através do exame micológico pré-tratamento, de infecção por Candida, obteve-se os 20 pacientes que constitui a real amostra que iniciou e terminou o tratamento, não incluindo nesse número os casos desistentes e os pacientes que apresentaram resultados micológicos pré-tratamento negativos.

A pesquisa foi aprovada pelo Comitê de Ética e Pesquisa da Universidade Estadual da Paraíba, seguindo todos os preceitos da resolução ${ }^{\circ}$ 196/96 do Conselho Nacional de Saúde.

A confirmação do diagnóstico clínico de candidose oral foi realizada através de análise micológica realizada no Laboratório de Micologia da Universidade Federal da Paraíba, em João Pessoa/PB. A partir do material coletado foi feita a visualização macroscópica da presença de hifas e pseudo-hifas. A coleta do material foi realizada utilizando-se Swabs estéreis e em seguida os mesmos foram inseridos em meio de cultura Ágar Sabouraud Dextrose, próprio para o crescimento da Candida. Foram realizados os exames de cultura, isolamento e identificação da espécie do gênero Candida conforme metodologia de rotina micológica e de acordo com o protocolo próprio do laboratório para 
Tabela 1. Representação dos resultados da sintomatologia clínica pós-tratamento.

\begin{tabular}{lccc}
\hline Sintomatologia & Ausente & Igual ao Inicial & Discreta Melhora \\
\hline Grupo-teste (Uncaria tomentosa) & 3 & 4 & 3 \\
Grupo-controle (Miconazol) & 4 & 3 & 3 \\
Total & 7 & 7 & 6 \\
\hline
\end{tabular}

$\mathrm{p}=0,6392 \rightarrow \mathrm{p}>0,05$.

fins confirmatórios. O cultivo foi realizado em Ágar Sabouraud Dextrose, adicionado de Cloranfenicol (100 $\mathrm{mg} / \mathrm{ml}$ ) em tubos de ensaio e, incubados a $37^{\circ} \mathrm{C}$ por 24 a 72 horas. Depois de verificado o crescimento da levedura, procedeu-se o isolamento, que foi realizado em placas de Petri, contendo Ágar Sabouraud mais Cloranfenicol pela técnica de esgotamento em estrias. As placas foram incubadas a $34{ }^{\circ} \mathrm{C}$ durante 24 horas. Realizou-se provas morfológicas e bioquímicas. Utilizou-se a chave de identificação de Kerger-Van Rij (1984), para chegar-se a identificação da levedura em estudo ao nível de espécie.

Após os resultados laboratoriais da análise micológica, os pacientes com diagnóstico positivo de candidose foram submetidos à terapia antifúngica. Os pacientes foram divididos em 02 grupos: no $1^{\circ}$ grupo, constituído por 10 pacientes, foi administrado o fitofármaco na forma de gel, em bisnagas contendo 10 g da Uncaria tomentosa (Imuno-Max), em quantidade suficiente para cobrir toda a área atingida, três vezes ao dia por um período de duas semanas, conforme indicações do fabricante. $\mathrm{O} 2^{\circ}$ grupo (controle-positivo) utilizou o Miconazol (Daktarin) a $4 \%$ na forma de gel, em bisnagas contendo $30 \mathrm{~g}$, três vezes ao dia e por duas semanas.

As técnicas estatísticas empregadas na análise dos dados foram: estatística descritiva e estatística inferencial (Teste de Correlação de Pearson).

\section{RESULTADOS E DISCUSSÃO}

As leveduras do gênero Candida são microorganismos integrantes da microbiota bucal do homem desde o nascimento. A densidade de espécies geralmente alta na cavidade oral e sendo, usualmente, isolada mais de uma espécie, dificultam a determinação do papel de cada uma no processo patológico e o efeito da terapia incidindo sobre cada espécie. No presente estudo, em cada amostra, foi identificada apenas a espécie dominante no meio, podendo existir mais de uma espécie em cada amostra.

Embasado na literatura pesquisada não foi possível encontrar nenhum estudo avaliando a atividade antifúngica da Uncaria tomentosa sobre o gênero
Candida na cavidade oral, ficando difícil confrontar os resultados aqui encontrados com os de outras pesquisas. Outro ponto que despertou interesse foi em relação aos sintomas, pois a maioria dos pacientes do presente estudo não apresentou a percepção subjetiva da sua patologia. Muitos não sabiam que apresentavam uma infecção fúngica na boca, uma vez que o diagnóstico, geralmente, fora realizado pelos cirurgiões-dentistas.

Quanto à prevalência das espécies do gênero Candida na cavidade oral, o exame micológico mostrou ser a Candida albicans a mais prevalente, com 73,91\% da amostra, seguida da Candida tropicalis $21,73 \%$ e por último, a Candida guilliermondii com 4,34\%.

A análise estatística foi realizada através do Teste de Correlação de Pearson. Não houve diferença significativa nas análises clínicas e laboratoriais, uma vez que o $p=0,6392$ na análise clínica e $p=0,6531$ na análise laboratorial foram maiores do que $\mathrm{p}>0,05$.

Devido ter sido a amostra pequena, os resultados estatísticos não puderam mostrar um nível de confiança acima de 95\%, o que ressalta a necessidade da continuidade deste estudo para obtenção de uma amostra superior a 30 pacientes.

Neste estudo observa-se que houve clinicamente, o desaparecimento da sintomatologia após 14 dias ter sido instituído o tratamento, em ambos os grupos. No grupo que utilizou o gel da Uncaria tomentosa (Imuno-Max/grupo-teste), 30\% (3) dos pacientes não apresentaram sintomatologia relacionada à candidose após os 14 dias de tratamento, enquanto que o grupo que utilizou o Miconazol (Daktarin/ grupo-controle) apresentou resultados praticamente semelhantes ao Imuno-Max Gel, onde a sintomatologia desapareceu em 40\% (4) dos pacientes. Esse fato revela a potente ação antifúngica e antiinflamatória da Uncaria tomentosa (Imuno-Max) frente às espécies de Candida encontradas. Observou-se, também, em alguns pacientes, melhoras discretas, onde três $(30 \%)$ eram pertencentes ao grupo-controle e três $(30 \%)$ ao grupoteste. Em ambos os grupos, 30\% (3) dos pacientes não apresentaram melhora na sintomatologia clínica, continuando com os mesmos sinais e sintomas do exame inicial (Tabela 1).

Entende-se que a avaliação clínica não deva ser

Tabela 2. Reações adversas aos fármacos apresentadas pelos pacientes.

\begin{tabular}{|c|c|c|}
\hline Reações adversas & $\mathrm{n}$ & $\%$ \\
\hline Sim & 4 & 20 \\
\hline Não & 16 & 80 \\
\hline Total & 20 & 100 \\
\hline
\end{tabular}


Tabela 3. Predominância de pacientes portadores de próteses dentais.

\begin{tabular}{lcc}
\hline Amostra quanto ao uso de próteses dentais & $\mathrm{n}$ & $\%$ \\
\hline Portador de prótese dental & 17 & 85 \\
Não-portador de prótese dental & 3 & 15 \\
Total & 20 & 100 \\
\hline
\end{tabular}

o melhor método de análise nos referidos casos, pois trata-se na sua grande maioria de pacientes portadores de próteses dentárias, ou seja, pacientes que muitas vezes apresentam estomatite protética. Logo, nos casos em que não houve melhora no quadro clínico pode-se traduzir na realidade em estomatites protéticas, que só desaparecem, em alguns casos, com a troca da prótese. Existiram pacientes que tiveram a avaliação clínica póstratamento ainda apresentando os mesmos sintomas iniciais e, no exame micológico pós-tratamento, obtevese resultado negativo, constando-se aí tratar-se de casos de estomatites protéticas, que podem apresentar o mesmo aspecto semiológico das infecções fúngicas.

Um dado importante na presente pesquisa é que nenhum paciente do grupo-teste referiu reações adversas no transcurso do tratamento, já sendo uma vantagem do fitofármaco sobre outros medicamentos que estão atualmente no mercado, uma vez que $4(40 \%)$ pacientes do grupo-controle relataram ter tido reações adversas no decorrer do tratamento. Três $(30 \%)$ relataram ter tido náuseas logo após a aplicação do produto nas áreas lesionadas e $1(10 \%)$ referiu dor epigástrica (Tabela 2$)$.

Neste estudo, grande parte dos pacientes com candidose oral são portadores de próteses dentárias $(85 \%)$ e destes $100 \%$ apresentaram estomatite protética (Tabela 3). Dos pacientes da presente pesquisa, apenas $3(15 \%)$ não eram portadores de próteses, sendo $2(10 \%)$ com sintomatologia de xerostomia e $1(5 \%)$ sem fatores causais evidentes.

Um ponto muito importante neste estudo é que, os pacientes portadores de próteses dentárias, foram orientados a realizar a remoção do aparelho protético antes de dormir e só colocá-los novamente ao acordar. Pesquisas futuras devem ter como foco de estudo a relação entre a real eficácia do fármaco $\mathrm{e} o$ ato de remoção da peça protética ao dormir, pois muitos dos casos clinicamente curados podem ser devidos ao ato de remoção da prótese, ficando o fármaco com papel secundário no tratamento. De acordo com Neville et al. (2004), muitas vezes, a colonização por Candida pode estar na peça protética e não na mucosa oral do paciente, o que não caracteriza uma infecção propriamente dita, já que os tecidos do hospedeiro não estão infectados.

$$
\text { Inúmeras pesquisas demonstram }
$$

predominância da espécie $C$. albicans em pacientes portadores de próteses dentárias, com xerostomia de etiologia variada, com AIDS, pacientes transplantados, com tumores malignos e com doença periodontal crônica. Neste estudo observou-se, também, uma elevada prevalência dessa espécie, o que vem a corroborar com a literatura (Oliveira et al., 1998; Ito et al., 2004; Menezes et al., 2006).

É de grande importância observar a ação farmacológica clínica e laboratorial da Uncaria tomentosa sobre várias espécies de Candida. Nesta pesquisa foi observada uma ação eficaz do fitofármaco sobre as espécies C. albicans, $C$. tropicalis e $C$. guilliermondii.

A Uncaria tomentosa é uma importante planta do ponto de vista fitoterápico, desde o início de seu uso, por índios da América do Sul, de forma profilática e terapêutica, a sua comercialização se expandiu pelo mundo. A caracterização de suas atividades biológicas é, portanto, seu papel principal (Jurgensen, 2005).

Acredita-se que o alto poder antiinflamatório da Uncaria tomentosa deva-se ao resultado da combinação de substâncias que atuam sinergicamente aumentando a atividade antiinflamatória (Heitzman et al., 2005).

O Miconazol (Daktarin Gel oral) nos últimos anos tem sido a droga de eleição no tratamento de candidoses orais, sendo este utilizado por via tópica. Torna bastante promissor os resultados aqui encontrados nesta pesquisa onde apresentou-se uma nova alternativa de tratamento para esta infecção.

Várias plantas têm sido estudadas, onde foram avaliadas suas propriedades antifúngicas, podendo citar a romã (Punica granatum Linn.), o extrato de própolis, melaleuca (Melaleuca alternifolia), que demonstram serem potentes no tratamento das infecções por Candida (Jandourek et al., 1998; Azevedo et al., 1999; Vasconcelos et al., 2006; Longhini et al., 2007).

Os resultados dos exames micológicos póstratamento mostraram uma melhor atuação da Uncaria tomentosa, uma vez que $60 \%$ (6) dos pacientes do grupoteste obtiveram resultados negativos no micológico pós-tratamento, enquanto que no grupo-controle (Miconazol), 50\% apresentaram resultados negativos a (Tabela 4).

Tabela 4. Representação dos resultados dos exames micológicos pós-tratamento.

\begin{tabular}{lcc}
\hline Exame micológico pós-tratamento & Positivo & Negativo \\
\hline Grupo-teste (Uncaria tomentosa) & 4 & 6 \\
Grupo-controle (Miconazol) & 5 & 5 \\
Total & 9 & 11 \\
\hline
\end{tabular}

$\mathrm{p}=0,6531 \rightarrow \mathrm{p}>0,05$ 
Os resultados, clinicamente, mostram uma eficácia terapêutica do Miconazol levemente superior a Uncaria tomentosa. Mas como já abordado anteriormente, os resultados clínicos não são o melhor método de se avaliar os presentes resultados. Logo, deve-se tomar como base os resultados laboratoriais.

Estudos futuros devem procurar obter uma planta de boa qualidade, selecionando aquelas que contenham essencialmente oxíndoles pentacíclicos, estes que promovem os reais efeitos antiinflamatórios e imunoestimulantes que se deseja no tratamento da Candidose oral.

\section{CONCLUSÃO}

A Candida albicans é a espécie mais prevalente nos pacientes com candidose oral. Diante dos resultados, observou-se que a Uncaria tomentosa demonstrou ter laboratorialmente uma eficácia terapêutica superior ao Miconazol, apesar de ter tido o Miconazol uma eficácia clinicamente melhor do que a Uncaria tomentosa. A Uncaria tomentosa revelou ser um fitofármaco promissor na Odontologia, no campo do tratamento antifúngico. A Uncaria tomentosa apresentou a vantagem sobre o Miconazol de não ter provocado reações adversas nos pacientes. Os pacientes usuários de próteses dentárias apresentaram uma maior prevalência e susceptibilidade ao desenvolvimento da candidose oral, apresentando clínico e laboratorialmente sinais positivos para a infecção. Estudos in vitro, para avaliar a atividade antifúngica de extratos da Uncaria tomentosa sobre espécies de Candida de forma isolada, deverão ser realizados, efetivando a eficácia dessa planta no tratamento da Candidose oral.

\section{REFERÊNCIAS}

Akesson C, Lindgren H, Pero RW, Leanderson T, Ivars F 2005. Quinic acid is a biologically active component of the Uncaria tomentosa extract C-Med 100. Int Immunopharmacol 5: 219-229.

Azevedo RVP, Komesu MC, Candido RC, Salvetti C, Rezende FHC 1999. Candida $\mathrm{sp}$ in the oral cavity with and without lesions: maximal inhibitory dilution of propolis and periogard. Rev Microbiol 30: 335-341.

Batista JM, Birman EG, Cury AE 1999. Suscetibilidade a antifúngicos de cepas de Candida albicans isoladas de pacientes com estomatite protética. Rev Odontol Univ São Paulo 13: 343-348.

Guggenheimer J, Moore P, Rossie K, Myers D, Mongelluzzzo MB, Bloqueie HM, Weyant R, Pomar T 2000. Insulindependent diabetes mellitus and oral soft tissue pathologies: Prevalence and characteristics of noncandidal lesions. Oral Surg Oral Pathol Oral Radiol Endodontol 89: 563-569.

Heitzman ME, Neto CC, Winiarz E, Vaisbergb AJ, Hammondc GM 2005. Ethnobotany, phytochemistry and pharmacology of Uncaria (Rubiaceae). Phytochemistry 66: 5-29.

Hunter KD, Gibson J, Lockhart P, Pithie A, Bagg J 1998. Fluconazole-resistant Candida species in the oral flora of fluconazole-exposed HID-positive patients. Oral Surg Oral Pathol Oral Radiol Endodontol 85: 558-564.

Ito YK, Martins CAP, Loberto JCS, Santos SSF, Jorge AOC 2004. In vitro antifungal susceptibility of Candida spp. isolates from patients with chronic periodontitis and from control patients. Braz Oral Res 18: 80-84.

Jandourek A, Vaishampayan, J, Vasquez J 1998. Effectiveness of the maleleuca oral solution for the treatment of fluconazole refractory oral candidiasis in patients with AIDS. AIDS 12: 1033-1037.

Jurgensen S, Dalboa S, Angersb P, Santos ARS, Ribeiro RM 2005. Involvement of 5-HT2 receptors in the antinociceptive effect of Uncaria tomentosa. Pharm Biochem Behav 81: 466-477.

Kleinegger C, Stoeckel D, Kurago Z 2001. A comparison of salivary calprotectin levels in subjects with and without oral candidiasis. Oral Surg Oral Pathol Oral Radiol Endodontol 92: 62-67.

Kloucek P, Polesny Z, Svobodobac B, Vlkovac E, Kokoskaa L 2005. Antibacterial screening of some Peruvian medicinal plants used in Callería District. $J$ Ethonopharmacol 99: 309-312.

Longhini R, Raksa SM, Oliveira ACP, Svidzinski TIE, Franco SL 2007. Obtenção de extratos de própolis sob diferentes condições e avaliação de sua atividade antifúngica. Rev Bras Farmacogn 17: 388-395.

Martino L, Martinot JLS, Franceschelli S, Leone A, Pizza C, Feo V 2006. Proapoptotic effect of Uncaria tomentosa extracts. J Ethnopharmacol 107: 91-94.

Menezes EA, Monteiro MNR, Parente TMC, Cunha FA, Augusto KL, Freire CCF 2006. Frequency and enzymatic activity of Candida albicans isolated from the oral cavity of HIV-positive patients at Fortaleza, Ceará. J Bras Patol Med Lab 42: 253-256.

Moore P, Guggenherimer J, Rossie K, Myers D, Mongelluzzo, MB, Bloqueie, H, Weyant R, Pomar T 2000. Insulindependent diabetes mellitus and oral soft tissue pathologies: Prevalence and characteries of Candida and candidal lesions. Oral Surg Oral Pathol Oral Radiol Endodontol 89: 570-576.

Neville BW, Damm DD, Allen CM, Bouquot JE 2004. Patologia oral e maxilofacial. $2^{\mathrm{a}}$ ed. Rio de Janeiro. Guanabara Koogan.

Oliveira EE, Silva SC, Soares AJ, Attux C, Cruvinel B, Silva MRR 1998. Toxinas killer e produção de enzimas por Candida albicans isoladas da mucosa bucal de pacientes com câncer. Rev Soc Bras Med Trop 31: 523-527.

Oliveira FQ, Gobira B, Guimarães C, Batista J, Barreto M, Souza M 2007. Espécies vegetais indicadas na odontologia. Rev Bras Farmacogn 17: 466-476.

Patton LL, Bonito A, Shugars D, Colina C 2001. A systematic review of the effectiveness of antifungal drugs for the prevention and treatment of oropharyngeal candidiasis in HIV-positive patients. Oral Surg Oral Pathol Oral Radiol Endodontol 92: 170-179.

Patton LL, Shugars AJ 2003. Which antifungal agent is most effective in oropharyngeal candidiasis? EvidenceBased Dentristy 4: 14-17.

Pilarski HZ, Skib DC, Gulewicz K 2006. Antioxidant activity of ethanolic and extracts of Uncaria tomentosa (Willd.) DC. J Ethonopharmacol 104: 18-23.

Radfar L, Shea Y, Fischer S, Sankar V, Leakan RA, Baum B, Pillemer S 2003. Fungal load and candidiasis in Sjogren's syndrome. Oral Surg Oral Pathol Oral Radiol Endodontol 96: 283-287.

Sheng Y, Akesson C, Holmgren K, Bryngelsson C, Giamapab 
V, Pero RW 2005. An active ingredient of Cat's Claw water extracts identification and efficacy of quinic acid. J Ethnopharmacol 96: 577-584.

Silva MSA, Silva MAR, Higino JS, Pereira MSV, Carvalho AAT 2008. Atividade antimicrobiana e antiaderente in vitro do extrato de Rosmarinus officinalis Linn. sobre bactérias orais planctônicas. Rev Bras Farmacogn 18: 236-240.

Taylor L 2002. Herbal Secreyts of the Rainflorest. 2a nd. Sage Press.

Vasconcelos LCS, Sampaio FC, Sampaio MCC, Pereira MSV, Higino JS, Peixoto MHP 2006. Minimum inhibitory concentration of adherence of Punica granatum Linn (pomegranate) gel against $S$. mutans, $S$. mitis and $C$. albicans. Braz Dent J 17: 223-227.

Vasconcelos KRF, Veiga Junior VF, Rocha WC, Bandeira MFCL 2008. Avaliação in vitro da atividade antibacteriana de um cimento odontológico à base de óleo-resina de Copaifera multijuga Hayne. Rev Bras Farmacogn 18 (Supl.): 733-738.

Vieira JDG, Ribeiro EL, Campos, CC, Pimenta FC, Toledo AO, Nagato GM, Souza NA, Ferreira WM 2005. Candida albicans isoladas da cavidade bucal de crianças com síndrome de Down: ocorrência e inibição do crescimento por Streptomyces sp. Rev Soc Bras Med Trop 38: 383-386. 